\title{
Notas en torno al desarrollo de regiones fronterizas en América Latina*
}

\author{
Punto y Raya
}

Entre tu pueblo y mi pueblo hay un punto $y$ una raya; la raya dice no hay paz, el punto via cerrada. $Y$ asi entre todos los pueblos raya y punto, punto $\mathrm{y}$ raya, con tantas rayas y puntos el mapa es un telegrama...

(del cancionero latinoamericano).

LA INTEGRACIÓN FÍSTCA: MEDIO Y FIN

Desde un punto de vista económico, el concepto de integxación de un grupo de Estados-naciones incluye cuatro tipos de elementos:

i) la interpenetración de mercados anteriormente separados por fronteras polf́ticas que restringen la circulación de mercaderías, personas y servicios entre los respectivos territorios nacionales;

ii) el aumento del grado de comunicación y de interdependencia de centros de decisión ubicados en esos territorios;

iii) la creación de un nuevo marco institucional para las relaciones entre los Estados que se integran. Ese marco institucional, sin prescindir de los Estados nacionales, se constituye progresivamente en un centro de decisiones compartidas por los mismos, pero con personalidad jurídica propia y cuya misión básica es compatibilizar las políticas y las decisiones de los centros nacionales con miras a maximizar el crecimiento y las ganancias del conjunto de países;

* Una versión reducida de este trabajo fue preparada como documento de apoyo del ILPES a la vi Conferencia de Ministros y Jefes de Organismos de Planificación de América Latina $y$ el Caribe, celebrada en La Habana, Cuba, en marzo de 1987. 
iv) la adaptación inducida o deliberada de las estructuràs productivas nacionales y de los flujos y prácticas de comercio a las nuevas condiciones del mercado integrado.

Como es obvio, la integración y cooperación económica entre países requiere como condición previa o como proceso paralelo, la articulación y el amplio desarrollo de la infraestructura de transportes y comunicaciones, particularmente en un sub-continente de la extensión de América Latina y el Caribe, caracterizado parcialmente precisamente por la precariedad de los sistemas de interconexión. En este sentido, la integración física, visualizada como integración de las redes de transportes y comunicaciones, constituye un medio instrumental para el logro de la integración económica. Pero también la integración física constituye un fin en sí misma en cuanto bajo tal denominación se incluye el aprovechamiento compartido de áreas de interés común, para lo cual dos o más países emprenden acciones concertadas de organización del espacio de dichas áreas.

En consecuencia, bajo el concepto de integración física queda incluida la integración de los sistemas de transportes y comunicaciones y también el manejo integrado de áreas de interés común. A su vez, dentro de este último concepto se incluyen dos modalidades de estrategias integracionistas: el manejo integrado de áreas de interés común para el aprovechamiento compartido de recursos, modalidad representada en la práctica por los programas de cuencas multinacionales para uso hídrico, energético, de navegación o de explotación de recuros y, por otro lado, el manejo integrado de áreas de interés común para la complementación del desarrollo regional, modalidad expresada por los programas de integración fronteriza.

La presencia de los organismos internacionales de cooperación técnica y financiera en estos campos ha sido importante y relativamente especializada.

El apoyo a los programas relacionados con las cuencas multinacionales en América Latina ha sido una cuestión preferente para la Organización de Estados Americanos (Departamento de Desarrollo Regional). Gabe hacer notar que América Latina posee algunas de las cuencas hidrográficas más importantes del mundo: la Cuenca Amazónica, la Cuenca del Orinoco, Ia Cuenca del Plata, la Cuenca del Lago Titicaca y la Cuenca del Golfo de Fonseca. El BID y el INTAL también han tenido una participación gravitante en estudios y proyectos sobre estas cuencas.

Los estudios y el financiamiento de proyectos de integración de sistemas de transportes y comunicaciones han sido apoyados principalmente por la CEPAL (División de Transportes) y por el BrD y el BIRF. La CAF hace parte del grupo cuatripartito CAF/JUNAC/BID/BIRF, que estudia los proyectos de transporte que contribuyen a la integración de los paises miembros del Acuerdo de Cartagena. 
El presente documento se centra exclusivamente en la cooperación y en la planificación para el desarrollo de regiones fronterizas, un tema en el cual el ILPes puede estar en mejores condiciones para ofrecer perspectivas adicionales, principalmente debido a las vinculaciones de este tema con el amplio campo del desarrollo regional (sub-nacional), un tópico en el cual el Instituto ha trabajado persistentemente durante los últimos años.

\section{EL SIGNIFICADO DE LAS AREAS FRONTERIZAS EN AMÉRIGA LATINA}

La cuestión fronteriza en América Latina y el Garibe tiene una dimensión concreta bastante mayor de lo que usualmente se piensa. Si se incluye la frontera entre México y Estados Unidos, existen 71 "posiciones de frontera" entre los 22 países considerados para estos efectos (países miembros del mpes, pertenecientes a la región y excluyendo los países que ocupan toda una isla).

Estas fronteras, verdaderas bisagras de integración, se extienden a lo largo de 70.000 kilómetros, cifra en la cual destacan los casos de Brasil con 12.303 kilómetros de frontera, Argentina con 9.389 kilómetros, Perú con 6.367, Bolivia con 6.340 y Ghile con 6.328 kilómetros. La frontera Argentino-Chilena por sí sola tiene una longitud de 5.818 kilómetros, siendo la tercera o cuarta frontera más extensa del mundo.

Considerando el primer nivel de la división política-administrativa en cada pás como unidad de referencia, se constata que las así definidas "áreas de frontera" incluyen uná superficie total igual a 21.819.670 $\mathrm{Km}^{2}$ (excluida la frontera entre México y los Estados Unidos de todos estos guarismos). Naturalmente esta es una cifra ilustrativa que incluye una contabilización múltiple debido a que muchas áreas fronterizas de varios países limitan simultáneamente con dos, tres o más áreas de países vecinos (por ejemplo, en el Brasil el Estado de Amazonas limita con Guyana, con Venezuela, con Colombia y con Perú).

Siempre desde un punto de vista de caracterización global de la situación, Ia población residente (cifra 1980) en todas las áreas de frontera suma 121.221.84I personas. Brasil, Chile $-y$ Argentina son los pafses con mayor población fronteriza, en tanto que Surinam, Belize y República Dominicana exhiben las cifras menores de población fronteriza. Nuevamente hay que señalar la contabilización múltiple y además el hecho de que no toda la población de una extensa área de frontera puede considerarse realmente como población "fronteriza".

También es de interés observar la situación atendiendo a la densidad de población en las áreas de frontera. Desde este punto de

$$
\left[\begin{array}{lll}
1 & 6 & 0
\end{array}\right]
$$


vista, El Salvador (142.78) y República Dominicana (123.45) aparecen muy por encima del resto de los paises; Paraguay (3.53), Venezuela (3.37), Perú (2.83), Guyana (2.42) y Surinam (0.62) muestran respectivamente los valores más bajos.

Naturalmente, Ias 71 "posiciones de frontera" tienen una importancia muy variada desde el punto de vista de la integración e interacción. Las fronteras más "vivas" son probablemente la mexicana-norteamericana, la colombo-venezolana, la boliviana-chilena pe ruana, la argentina-chilena, la argentina-uruguaya y la brasileñaparagua, entre otras.

\section{NATURALEZA DE LAS AREAS Y REGTONES FRONTERIZAS}

Cabe observar primeramente la cuestión de las áreas fronterizas desde un punto de vista puramente geográfico. En tal contexto, el término área fronteriza alude única y exclusivamente a una situación locacional dada por el carácter de limítrofe que tales áreas tienen con países vecinos. No tiene otra connotación y asimismo no tiene contenido valórico desde el punto de vista económico. En este mismo plano es útil recordar que muchos países simplemente no tienen áreas de frontera (la mayor parte de los países isleños por ejemplo) en tanto que en otros todas las regiones son áreas de fronteras, como es el caso de Chile.

Desde un punto de vista económico y social el concepto ahora de región fronteriza, adquiere complejidades adicionales, ya que en este contexto estas regiones deben ser conceptualizadas como espacios subnacionales limitrofes a paises vecinos, en los cuales se manifiestan formas particulares de relación y superposición de dos (o más) sistemas (o estilos) económicos y de dos (o más) modelos diferentes de politica económica.

Los antecedentes últimos de esta forma de conceptualizar a las regiones fronterizas se encuentran en la famosa clase dictada por $\mathrm{F}$. Perroux en la Universidad de Harvard en 1949, en la que planteó su crítica al concepto de espacio "banal" o geonómico e introdujo Ios varios conceptos de espacio económico. Aun cuando la conferencia no estaba dirigida al tema fronterizo como tal, Perroux se esforzó en mostrar - con referencia al caso europeo- la superior potencialidad analítica y política del concepto de espacio económico por sobre la noción de espacio geográfico y la importancia de ello en relación a un proyecto de una Europa unida, con "fronteras devaluadas", para utilizar su misma terminología ${ }^{1}$.

珜. Perroux, "Economic Space: Theory and Application" en: J. Friedmann y W. Alonso (eds.), ,Regional Development and Planning (Cambridge: The Mri Press, 1964). 
En general, el desarroilo de regiones fronterizas ha sido un tenia que históricamente ha llamado la atención de los especialistas en la teoría de la localización y en la teoría de los polos de crecimiento, aunque el tratamiento dado al tema ha destacado las desventajas que tales regiones experimentan, debido al hecho de que los límites políticos representan barreras artificiales a una organización económica racional de áreas potencialmente complementarias y también debido a que tanto el sector público como el sector privado tienden a evitar la inversión en áreas en las cuales pueden surgir conflictos. Tanto Christaller como Losch, pioneros de la moderna teoría de la localización, han sostenido que los objetivos políticos del EstadoNación serian perjudiciales para las regiones fronterizas. Losch por ejemplo, sostuvo que el principal objetivo económico era la prosperidad, seguido en orden de prioridad, por la cultura, el poder $y$, la permanencia o integralidad; en contraste, los objetivos del EstadoNación siguen un orden exactamente inverso ${ }^{2}$. Christaller sostuvo que las áreas fronterizas quedan con toda probabilidad sujetas al "principio de separación", en que "la autoridad de la voluntad soberana y establecida" tiene precedencia sobre la "racionalidad de los principios económicos"3. Los teóricos de los polos de crecimiento que se han ocupado de las cuestiones vinculadas a las regiones de frontera también han enfatizado los conflictos translimítrofes ${ }^{4}$ quizás bajo la influencia de la situación de las regiones francesas limitrofes con Alemania.

Las distorsiones que los límites internacionales imponen sobre el "paisaje económico" óptimo han constituido la temática preferente de Ios analistas, como lo señalara Melchior:

"En general, la existencia de barreras al comercio determinará que la localización de la industria se oriente más en función de los mercados internos, tendiendo a desplazarse hacia puntos más distantes de la frontera que en el caso de la consideración de los mercados exteriores. Esto es denominado por Giersch como fenómeno de aglomeración nacional, que resulta de la acción de las fronteras erigidas como obstáculos al flujo internacional de las bienes.

Se ha señalado con razón que la aglomeración nacional, provocada por la existencia de barreras arancelarias, restricciones al 1954).

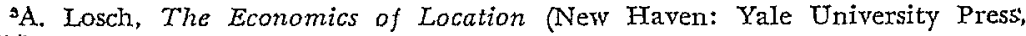

${ }^{3}$ W. Christaller, Central Places in Southern Germany (New Jersey: Englewood. Cliffs, 1966).

'J. Boudeville, "Analyse économique des régions frontières", Economie et Societés, 5, Paris, I971.

R. Gendarme, "Les problèmes économiques des régions frontières européennes", Revue économique, 21, Parfs, 1970. 
comercio, fronteras políticas, etc., ha actuado en contraposición a la tendencia a la aglomeración internacional que, en un caso ideal, se produciría sobre un espacio no afectado por la presencia distorsionante de las fronteras"

Volviendo entonces al concepto de región fronteriza elaborado en función de consideraciones económicas y sociales, corresponde senalar que una situación fronteriza pasa a convertirse en un "problema" especifico de la política pública cuando la mencionada interacción de los dos (o a veces más) sistemas y modelos de política económica opera asimétricamente, con un inequitativo reparto entre las regiones vecinas de los costos y beneficios de la propia interacción y del efecto de las políticas económicas. Esto se puede ilustrar alud:endo, por ejemplo, al caso de la frontera Colombo-Venezolana y a la situación creada en ella por la devaluación de la moneda renezolana en 1983, una medida de política económica que trastocó por completo el sistema binacional de intercambio inter-regional fronterizo, asumiendo Colombia $\mathrm{y}$ en particular el Departamento Norte de Santander los costos del ajuste.

Se deduce en consecuencia que en virtud del carácter de manifestación espacialmente localizada de la interacción de dos (o más) sistemas y principalmente de dos (o más) modelos de política económica, el tratamiento de una situación fronteriza "problemática" depende fuertemente de las modalidades de relaciones globales entre las conomías vecinas y de concebir a las fronteras más bien como espacios de cooperación, contacto y aproximación que como barreras polfticas y económicas; esto último cubone a su vez estimular la participación conjunta de las poblaciones en la búsqueda de soluciones armónicas en vez de fomentar antagonismos muchas reces artificiales que desconocen por lo demás el conciderable grado de fluidez e interacción social que está presente en las reg:ones fronterizas con escasas barreras naturales al intercambio.

Hay, por supuesto, otras viciones sobre la cuestión fronteriza, como por ejemplo, aquellas encuadradas en concepciones geopoliticas y de seguridad nacional. En esos contextos, las regiones fronterizas son espacios de confrontación $\mathrm{v}$ por tanto carecen ecas visiones de interés en el plano de esta discusión.

EI DESARRollo FRONTERIZO COMO CASO ESPECTAT. DEL MODELO GENERAL DE DESARROLLO REGIONAL

Si se revisa la literatura más actualizada sobre el problema del desarrollo de regiones fronterizas, aparece con claridad un hecho: la

FE. Melchior, "Fronteras internacionales y espacios económicos", ILPES, Documento CPRD-C/33, Santiago de Chile, 1981. 
mayor parte de los autores coincide en considerar el desarrollo fronterizo como un problema típico de desarrollo regional (subnacional). Por ejemplo:

"En tal perspectiva, las áreas de frontera y sus problemas, se presentan como componentes y resultantes de los procesos globales de desarrollo regional"'B.

"El enfoque 'regional' de la complementac'ón económica binacional y de la integración física puede ser menos espectacular políticamente y aún exhibir una fisonomía fragmentaria, pero emana más establemente de una realidad que suele ser bastante distinta según de qué regiones específicas se trata"7.

“... el contexto del presente artículo, el que trata acerca de los problemas del desarrollo y de la formulación de políticas en regiones vecinas separadas por límites internacionales"8.

Esta posición también ha sido la posición del ILPEs:

"... el desarrollo de las regiones fronterizas no es sino un caso especial del 'caso general del desarrollo regional"',

y también de lá propia CEPAL, expresada en el Informe sobre el Seminario sobre Integración y Desarrollo Regional en la Patagonia Argentino-Chilena, celebrado en 1985 en la ciudad de Punta Arenas:

"El aprovechamiento de las potencialidades económicas y sociales no depende sólo de que se reciba desde afuera, sino sobre todo de lo que la región puede hacer por sí misma; por ejemplo, en relación con su capacidad para aprovechar su propio ambiente, crear recursos y aprender a explotarlos conforme a sus condiciones específicas. Ello supone la movilización de la creatividad, iniciativa, capacidad organizativa y otras fuerzas propias de los pobladores de la región, entre las que se cuentan su voluntad y experiencia integradoras"10.

Una característica común a casi todos los trabajos recién citados es su falta de especificación en términos de la modalidad de desarrollo regional que incluiría dentro de sí el desarrollo fronterizo. En. tal sentido se detecta una carencia de estudios conceptuales que per-

Instituto Internacional de Integración, Convenio Andrés Bello, La integración fronteriza en la subregión andina (La Paz: 1985).

T. Barros Ch., Cooperación económica chileno-argentina: evaluación histórica (Santiago: Universidad de Chile, Instituto de Estudios Internacionales, 1986).

${ }^{8} \mathrm{~N}$. Hansen, "International Cooperation in Border Regions. An Overview and Research Agenda", International Regional Science Review, Vol. 8, No 3, 1983.

"ILPES, "Colombia: Observaciones en torno a la propuesta de un plan de desarrollo frónterizo". Informe de una misión de asesoria al DNP (Colómbia), 1985.

${ }^{10}$ CEPAL (1985), Documento LC/G.1390, 26/12/85. 
Sergio Boisier / Notas en torno al desarrollo de regiones fronterizas...

mitan ligar de una manera práctica y concreta la política de desarrollo fronterizo con la política nacional de desarrollo regional de cada pais.

A idéntica conclusión llega el Instituto Internacional de Integración:

"Los estudios sobre la integración fronteriza deben estar concentrados en la formulación de un cuerpo de conceptos básicos sobre la caracterización y el rol de las áreas de frontera, así como de una metodología propia y adecuada para la investigación de la sociedad y la economía de esas áreas. La definición de programas y politicas adoptadas a dichos fines debe permitir incorporar, cada vez más explícitamente, los instrumentos y mecanismos de los sistemas latinoamericanos de integración y cooperación económica, con la finalidad de conducir e implementar, a través de ellas, las relaciones existentes entre las áreas limítrofes y promover otras nuevas a fin de fortalecer y ampliar las acciones nacionales de desarrollo e integración"11.

En este contexto, entonces, una pregunta fundamental se relaciona con la concepción específica sobre desarrollo regional y sobre la planificación de dicho proceso a la cual debe recurrirse para un adecuado tratamiento de la cuestión fronteriza. ¿Será una concepción traclicronal (y ciertamente todavía dominante) del fenómeno del desarrollo regional suficiente para el propósito señalado? Si ello no fuese asi habria entonces que recurrir a interpretaciones alternativas, de mayor amplitud y con capacidad para manejar simultáneamente varias dimensiones del proceso de desarrollo regional.

La concepción que ha dominado ja práctica de la planificación del desarrollo regional desde la década de los cuarenta hasta prácticamente hoy día, queda bien representada por una de las definiciones de ella de más amplia aceptación tradicional: "Como tal, la politica (de desarrollo) regional es un componente de la planificación nacional, preocupada con asignar recursos entre regiones"12. Este tipo de enfoque, predominante en la literatura y en la práctica es sumamente restringido, puesto que reduce el problema del desarrollo regional a un solo factor causal: los recursos asignados a cada región y pasa por alto -en relación a las regiones fronterizas- otro elemento muchas veces determinante de su desarrollo, como es el impacto de las polfticas macroeconómicas del pafs vecino y configura además una situación de clara centralización en el manejo de la cuestión regional, puesto que la asignación de recursos entre regio-

1Instituto Internacional de Integración, op. cit., pp. 103-104

19. Alden y $\mathrm{R}$. Morgan, Regional Planning: A comprehensive View (London: Lconard Hill Books, 1974). 
nes es, teórica y empíricamente, un proceso por definición centralizado en tanto que la descentralización por su lado aparece también como una cuestión importante en el manejo de las regiones de frontera.

Ast pues, parece imprescindible acudir a otro tipo de interpretación del fenómeno del desarrollo regional y en este contexto, el enfoque desarrollado en el ILPES, parece particularmente apropiado.

Si se reflexiona sobre el fenómeno del desarrollo regional a largo plazo y si se buscan factores con real capacidad de explicar la existencia o la falta de dicho proceso, entonces pueden adelantarse hipótesis como las siguientes:

- el crecimiento económico regional parece depender tanto de la cuantía de recursos (naturales) poseída por una región como principalmente del monto de recursos nacionales (económicos) que la región puede captar mediante diversos mecanismos;

- el crecimiento económico regional también parece depender estrechamente del impacto sobre la región del cuadro global de la política económica, que en algunos casos puede ser un elemento que se sume al efecto derivado del monto de recursos, en tanto que en otros puede jugar en sentido inverso, llegando a anular el efecto crecimiento de la inversión de recursos;

- el desarrollo regional (como fenómeno cualitativamente distinto del crecimiento) parece estar más ligado a procesos endógenos de la región que se asocian a cuestiones tales como: i) el incremento paulatino de la capacidad de decisión propia de la región (lo que a su turno depende de su estructura política, de su clase política, de su tecnocracia, de la posibilidad de reemplazar la confrontación social por la concertación en torno a proyectos políticos regionales, etc.); ii) el logro de la sustentabilidad del crecimiento, que se vincula con las formas de apropiación y de inversión local del excedente, en busca de una creciente diversificación productiva y del reemplazo de recursos no renovables (estando ello a su vez asociado al contenido regional de la política fiscal, a la existencia de un empresariado regional y a la propia capacidad de negociación regional); iii) crecientes niveles de inclusión social, tanto en términos del reparto del ingreso como en términos de participación (política) de la población (cuestión que a su vez depende de complejos elementos sociales e institucionales tanto formales como informales); $\mathrm{y}, \mathrm{fi}-$ nalmente, iv) una actitud colectiva de preservación del medio ambiente y de los recursos en general. No resulta impropio señalar entonces que el diesarrollo regional tiene mucho que ver con la capacidad de "organización social" de las regiones ${ }^{13}$.

${ }^{13}$ S. Boisier, "Política económica, organización social y desarrollo regional", Cuaderno No 29, ILPES, Santiago de Chile, 1982. 
Sergio Boisier / Notas en torno al desarrollo de regiones fronterizas...

A la luz de la presentación anterior, la planificación regional puede ser entendida como una actividad que contiene y se expresa simultáneamente en tres funciones: primero, una función de asignación, asociada a los criterios y procedimientos para repartir los recursos entre las regiones, incluyendo el diseño de los mecanismos de transferencia; segundo, una función de compensación asociada al análisis y a los procedimientos de negociación dirigidos a compensar el efecto detrimental sobre las regiones (sobre algunas de ellas) del cuadro de la política económica tanto macro como sectorial; y, tercero, una función de activación asociada a la creación y estímulo de un complejo conjunto de instituciones políticas, burocráticas y sociales que sean funcionales a la transformación del crecimiento en desarrollo y que configurarán, en definitiva, la comunidad regional organizada.

Es importante agregar que la primera de estas funciones es por cierto de naturaleza económica, en buena medida exógena a la región y considerablemente centralizada en su manejo; la segunda función es de naturaleza política principalmente, también exógena a la región y desconcentrada desde el punto de vista de su ejecución; por último, la tercera función es de naturaleza sociológica, endógena a la región y descentralizada por completo. En síntesis, este "modelo" de planificación regional tiende a destacar el papel diferenciado pero complementario de los dos más importantes actores del desarrollo regional: el Estado por un lado, en su doble papel de agente directo como formulador de políticas explícitas de crecimiento regional $e$ indirecto como formulador y ejecutor del cuadro de política económica nacional, y la región por otro, no ya como simple configuración geográfica, sino como comunidad organizada, locacionalmente específica y dotada de un sentimiento colectivo de pertenencia regional.

¿Gómo se insertan entonces las regiones fronterizas en este marco conceptual? No parecen escapar a los límites del modelo general, salvo el hecho de que en este tipo de regiones aparecen dos sub-factores de crecimiento singularmente importantes: primero, su generalmente bajo grado de articulación física y económica con las regiones centrales dinámicas de su propio país (en tal sentido se trata de regiones periféricas y excéntricas); segundo, el impacto de la política económica global (fiscal, monetaria, cambiaria, etc.) corresponde tanto al cuadro nacional como al cuadro extranacional (del país vecino). Este es el elemento que hace verdaderamente complicado el problema, puesto que las decisiones correspondientes no sólo son exógenas a la región jsino que también lo son al país! En lo demás, las diferencias entre las regiones fronterizas y el resto son más cuantitativas que cualitativas.

La argumentación precedente pone de relieve que el desarrallo 
de algunas regiones fronterizas no es solamente un problema enmarcado en el cuadro del desarrollo regional sino también un problema cuya solución se encuentra en el marco de la política económica nacional y de los acuerdos bilaterales de integración o de coordinación de políticas. En términos de bagaje profesional son, tanto los especialistas en comercio internacional en general y en política cambiaria en particular como los especialistas en desarrollo regional, quienes están equipados para proponer soluciones.

A fin de establecer una mayor especificidad del "caso especial" que correspondería al desarrollo de las regiones fronterizas hay que examinar los objetivos que, en el modelo general, son planteados para una región cualquiera. No significa ello que todos los objetivos del modelo general sean igualmente aplicables a cada una de las regiones; precisamente un papel de una estrategia nacional de desarrollo regional es ponderar este conjunto de objetivos en cada región.

Son básicamente seis los objetivos regionales usuales. De su peso relativo dependerá el logro de situaciones de crecimiento económico, de desarrollo, de mero soporte, etc. Cada objetivo está ligado por supuesto, a un conjunto de elementos condicionantes o si se quiere, a un conjunto de factores causales. Estos objetivos y sus correspondientes condicionantes se enumeran a continuación:

i) Crecimiento económioo, entendido como un aumento significativo en la capapcidad de producción de bienes y servicios de la región. El logro de un objetivo como el descrito depende de la situación existente en relación a:

i.1) la base de recursos regionales, tanto naturales como humanos y de la relación tecnológica entre ellos; cional;

i.2) la articulación física y económica con la economfa na-

i.3) el monto de recursos económicos (financieros) propios de la región y nacionales, captados por la región;

i.4) el impacto de la política macroeconómica tanto nacional como extra-nacional en el caso de las regiones de frontera;

i.5) la inserción en el mercado internacional, incluyendo el mercado fronterizo, a partir de la exportación significativa de recursos, bienes o servicios.

ii) Sustentabilidad del crecimiento económico, entendida como la permanencia a largo plazo del incremento de la capacidad de producción regional. Este objetivo depende de:

ii.1) Ia captación y reinversión regional del excedente econó- 
mico, cuestión que a su vez está condicionada por: a) la polftica tributaria y la politica de gasto fiscal en su dimensión regional; b) la existencia y pautas de conducta de un empresariado local comprometido con el desarrollo regional;

ii.2) la diversificación económica progresiya de la estructura productiva regional, lo que a su vez se asocia a asuntos como: a) posibilidades reales de la diversificación, incluyendo acuerdos binacionales en el caso de las regiones de frontera; b) existencia de una cartera de proyectos de inversión en permanente actualización.

iii) Equilibrio espacial, entendido como un reparto armónico de actividades sobre el espacio regional (equivale al concepto de integración intrarregional), objetivo que depende de:

iii.1) el patrón sectorial de crecimiento elegido, puesto que sectores como mineria e industria tienden a producir concentraciones espaciales, en tanto que agricultura o turismo producen efectos dispersores;

iii.2) la política de construcción de infraestructura;

iii.3) las caracteristicas de la política urbana en la región.

iv) Autonomia regional, entendida como una creciente capacidad de la región para optar por estilos de desarrollo y para ejecutar su propio desarrollo, un objetivo cuyo logro es función de:

iv.l) el grado de descentralización y desconcentración territorial prevaleciente en el nivel nacional;

iv.2) la estructura política de la región y forma de funcionamiento de ella, incluyendo la calidad de la "clase política" regional;

iv.3) la estructura administrativa regional, incluyendo el propio sistema de planificación y la calidad de los cuadros técnicos;

iv.4) la particular forma de organización social de la comunidad regional, incluyendo dentro de este concepto la "densidad" del tejido social, las tradiciones y formas de participación y la percepción colectiva de los problemas regionales;

iv.5) el nivel de conflicto o de concertación social regional y la capacidad para subordinar los intereses de grupo a un interés colectivo.

v) Inclusión sacial, entendida como equidad en el reparto del producto económico (distribución del ingreso y acceso a servicios sociales) y como participación en la propia planificación, y en otros procesos polfticos. Un objetivo como éste depende de:

v.I) el gasto público en la prestación de servicios sociales; 
v.2) el patrón sectorial de crecimiento elegido, por consideraciones funcionales similares a las expresadas en el punto iii.1) supra;

v.3) la organización social de la comunidad, en particular en relación a la existencia de instituciones públicas o no, capaces de articular los sectores informales regionales con el sector formal, tanto en el plano económico como en el plano socio-institucional.

vi) Protección del medio ambiente y de los recursos, un objetivo que se asocia a:

vi.1) la educación, es decir, al contenido de "valores" medioambientalista en los programas educativos de todo nivel;

vi.2) Ia investigación cientifica y tecnológica de los eco-sistemas correspondientes, con miras a su utilización racional;

vi.3) la existencia de normas, premios y castigos en relación a la explotación y uso de recursos.

Como se indicó, este conjunto genérico de objetivos debe ser. ponderado en cada región, de tal forma que, para utilizar un símil matemático, el "estado" que se desea alcanzar en una región (simple crecimiento, desarrollo, etc.) resulta en una especie de función lineal convexa del conjunto de objetivos. Esto es como se dijo, una función importante contenida en el diseño de una estrategia nacional de desarrollo regional.

Establecida esta suerte de función para una región fronteriza, cl logro del "estado" expresado por la función aludida puede obtenerse a su vez mediante dos estrategias alternativas. La primera de ella; (Estrategia A) consiste en un mix de políticas para alcanzar el "estado" establecido sin oonsiderar las posibilidades de una integración supra-nacional; la segunda estrategia (Estrategia B) consiste, inversamente, en un mix de políticas teniendo presente un marco de integración supra-nacional para ellas.

La secuencia entonces del procedimiento de planificación para una región fronteriza puede ser simplificadamente reducida a los pasos siguientes:

i) en la estrategia nacional de desarrollo regional debe especificarse el "estado" deseable a lograrse en la región (esto, por supuesto, definirá simultáneamente el papel de la región en dica estrategia);

ii) a la luz de otras consideraciones (de orden internacional principalmente) debe optarse por una estrategia especifica para la región (con integración supra-nacional o sin ella);

iii) elegida la estrategia, debe identificarse el mix de políticas en 
Sergio Boisier / Notas en torno al desarrollo de regiones fronterizas...

función de los factores condicionantes del conjunto de objetivos genéricos;

iv) dependiendo de la estrategia elegida, debe prepararse un calendario y un programa de acuerdos internacionales.

Este esquema se resume en el cuadro siguiente, el cual se muestra sólo como una ilustración, puesto que la importancia relativa de cada elemento solamente puede ser precisada en situaciones reales.

La estrategia A (sin integración) presupone el uso de políticas regionales y nacionales que tienden a "defender" a la región de.los efectos probables de la politica económica extra-nacionalit. Es en cierto sentido, una estrategia reactiva si se la compara con la estrategia $\mathrm{B}$, mediante la cual se busca aprovechar el establecimiento de un espacio ampliado de circulación económica a través de acuerdos de cooperación supra-nacional.

La creación de un espacio económico ampliado -implícito en la estrategia B- tiene implicaciones no sólo en el campo de las polfticas nacionales sino también en el campo más amplio de las decisiones internacionales. En relación a la frontera Colombo-Venezolana se ha planteado justamente esto:

"A estos fines los programas fronterizos promoverían la consolidación de centros a través de los cuales se incremente y difunda el progreso económico y social, se descentralice el financiamiento público, las inversiones y la toma de decisiones, y se incremente la participación local en todas las etapas de ese proceso.

Esta estrategia se basa, por consiguiente, en la movilización tanto de los recursos materiales y sociales del espacio fronterizo y del país respectivo en la medida en que estos últimos entran a formar parte de las relaciones de producción, como de los otros factores que por la vía de aquellas articulaciones fronterizas se incorporan a ese acerbo. Lo antes dicho significa que en mucha medida la viabilidad del desarrollo de esas regiones y el efectivo cumplimiento del nuevo rol que se les asigna, no dependerá sólo de las fuerzas económicas y sociales propias de esas áreas y de las políticas y programas nacionales y regionales que las impulsan, sino también de las provenientes de la región y del país limítrofe, de la política global y regional de éste y, de-de luego, de los mecanismos binacionales y multilaterales de cooperación y desarrollo que inciden sobre la evolución de esas zonas.

Desde esta perspectiva, en la que se pone de relieve el papel que desempeñan los factores y las interacciones económicas y so-

\footnotetext{
thor ejemplo, la paulatina devaluación del peso colombiano ha tenido como efecto lateral "compensar" al Departamento Norte Santander del impacto de la devaluación venezolana de 1983.
} 


\section{IMPORTANCIA RELATIVA DE FACTORES CONDICIONANTES \\ DE LOS OBJETIVOS REGIONALES EN CADA ESTRATEGIA FRONTERIZA}

\begin{tabular}{|c|c|c|c|}
\hline \multirow{2}{*}{$\begin{array}{l}\text { OBJETIVOS } \\
\text { REGIONALES } \\
\text { GENERICOS }\end{array}$} & \multirow[b]{2}{*}{ FACTORES GONDICIONANTES } & \multicolumn{2}{|c|}{ ESTRATEGIA } \\
\hline & & $\begin{array}{c}\text { A } \\
\text { (reactiva) }\end{array}$ & $\begin{array}{c}\text { B } \\
\text { (integración) }\end{array}$ \\
\hline $\begin{array}{l}\text { Grecimiento } \\
\text { Económico }\end{array}$ & $\begin{array}{l}\text { - Base de recursos regionales } \\
\text { - Articulación física con } \\
\text { economia(s) nacional(es) } \\
\text { - Monto de recursos económicos } \\
\text { - Impacto de polftica macro. } \\
\text { nacional y extra-nacional } \\
\text { - Inserción en mercados } \\
\text { externos }\end{array}$ & $\begin{array}{l}A>B \\
A>B\end{array}$ & $\begin{array}{l}B>A \\
B>A \\
B>A\end{array}$ \\
\hline Sustentabilidad & $\begin{array}{l}\text { - Captación y reinversión del } \\
\text { excedente } \\
\text { - Diversificación económica }\end{array}$ & $\begin{array}{l}A>B \\
A>B\end{array}$ & \\
\hline $\begin{array}{l}\text { Equilibrio } \\
\text { espacial }\end{array}$ & $\begin{array}{l}\text { - Patrón sectorial de crecimiento } \\
\text { - Política de infraestructura } \\
\text { - Política urbana }\end{array}$ & & $\begin{array}{l}B>A \\
B>A \\
B>A\end{array}$ \\
\hline $\begin{array}{l}\text { Autonomfa } \\
\text { regional }\end{array}$ & $\begin{array}{l}\text { - Grado de descentralización } \\
\text { - Estructura política } \\
\text { - Estructura administrativa } \\
\text { - Organización social } \\
\text { - Nivel de concertación }\end{array}$ & & $\begin{array}{l}B>A \\
B>A \\
B>A \\
B>A \\
B>A\end{array}$ \\
\hline $\begin{array}{l}\text { Inclusión } \\
\text { social }\end{array}$ & $\begin{array}{l}\text { - Gasto público en servicios } \\
\text { - Patrón sectorial de } \\
\text { crecimiento } \\
\text { - Organización social }\end{array}$ & $\begin{array}{l}A>B \\
A>B\end{array}$ & $B>A$ \\
\hline $\begin{array}{l}\text { Protección } \\
\text { del medio }\end{array}$ & $\begin{array}{l}\text { - Educación } \\
\text { - Investigación G y T } \\
\text { - Normas }\end{array}$ & $\begin{array}{l}A=B \\
A=B \\
A=B\end{array}$ & $\begin{array}{l}B=A \\
B=A \\
B=A\end{array}$ \\
\hline
\end{tabular}

ciales fronterizas en el desarrollo de estas áreas, aparece más clara la necesidad de ampliar la dimensión y dinámica del concepto generalmente prevaleciente del espacio fronterizo, con miras a darle cabida a todos los elementos que constituyen la base objetiva de la "realidad fronteriza" y a partir de ésta trazar, en el 
Sergio Boisicr / Notas en torno al desarrollo de regiones fronterizas...

marco de un proceso de planificación, las líneas maestras de la estrategia y de las polfticas y programas de desarrollo fronterizo y definir las acciones para llegar a los objetivos que se adopten.

De este modo podrán inducirse hacia la cristalización de las metas antes mencionadas los recursos productivos existentes a uno y otro lado de la frontera, ampliando las naturales facilidades para su movilización y aprovechando con iguales fines las ventajas de la localización geográfica de dichas áreas, la potencialidad de esas interacciones y la coincidencia de intereses y capacidades de la sociedad fronteriza frente a los problemas de su desarrollo"15.

En resumen, el control del desarrollo de las regiones fronterizas puede ser enmarcado perfectamente dentro del "modelo" general de planificación regional, debido principalmente a dos argumentos:

i) al destacar Ios factores más relevantes que pueden explicar los diversos "estados" de desarrollo de una región de fronteras, éstos coinciden con los factores pertenecientes al modelo general, con la importante consideración adicional en referencia al grado de articulación de la economía fronteriza con la propia economía nacional y en referencia al doble impacto que las regiones fronterizas puedan recibir por parte de la política macroeconómica nacional, así como por parte de la política macroeconómica del país vecino;

ii) desde el punto de vista de la planificación del desarrollo de tales regiones, el paso inicial se inscribe precisamente dentro de la formulación de la estrategia nacional de desarrollo regional, como se indicó más atrás; asimismo, la identificación del mix de políticas que darán expresión concreta a la estrategia específica para la reg:ón fronteriza no escapa del cuadro genérico aplicado a la planifi. cación de cualquier tipo de región.

En consecuencia, tanto desde el punto de vista de una teoria sustantiva de la planificación de regiones fronterizas, así como desde el punto de vista de una teoria de procedimiento de tal planificación, el control de desarrollo fronterizo se ubica principalmente dentro de una versión comprehensiva y actualizada acerca de la naturaleza del proceso de desarrollo regional y de su planificación.

PostbILIDADES Y LIMITACrones de LA Acctón CONJUNTA EN REGIONES FRONTERIZAS

El potencial de desarrollo cooperativo internacional representado por las regiones fronterizas en América Latina y el Caribe es consi-

${ }^{1 \gamma}$ A. Power, "Desarrollo fronterizo y proyectos de inversión", Comercio Exterior, Vol. 15, No 11, Instituto Colombiano de Comercio Exterior, Bogotá, 1982. 
derablemente elevado, si se juzga por la magnitud geográfica, demográfica y económica de dichas regiones, pero las dificultades no son menores en modo alguno. La cuestión es, como lo señala Aragao, transformar la región que se extiende como periferia de una frontera en un fecundo campo de experimentación de un esfuerzo cooperativo entre naciones y grupo sociales distintos asociados en la búsqueida de objetivos comunes ${ }^{16}$. A tal propósito deben apuntar acciones concretas, algunas de las cuales son identificadas por eI Instituto Internacional de Integración en la forma siguiente:

"La experiencia lograda en los diversos programas de integración fronteriza ejecutados hasta Ia fecha y particularmente los emprendidos en las fronteras colombo-venezolana y colomboecuatoriana, permiten detectar algunos aspectos centrales que, aparte de la asignación de recursos para usos alternativos, son de gran importancia operativa para los propósitos de la integración fronteriza. Entre éstos se destacan los aspectos cambiarios, los mecanismos de promoción, la reglamentación del tráfico fronterizo y la posibilidad de inversiones multinacionales.

\section{a) Aspectos institucionales y administrativos}

En primer término se requiere una adecuación de los instrumentos de decisión, de estudio y de ejecución - a nivel nacional y regional-a los objetivos del programa de integración fronteriza; las sugerencias contenidas en el capítulo sobre los aspectos institucionales del proceso, son completamente pertinentes sobre el particular. Si bien, el éxito de los programas de integración fronteriza está asociado a un efectivo compromiso de la voluntad política de los gogiernos interesados, es del todo evidente que el impulso vital de esos programas está localizado en la propia región a la que se destinan, por lo que deben ser incorporadas al programa todas las fuerzas dinámicas de la economía regional.

La necesidad de que el órgano conductor del proceso esté dotado de la necesaria autonomía financiera y administrativa, debe complementarse con la participación y el acceso en su directorio a los sectores sociales y económicos más representativos de la región, permitiéndoles ejercer la dirección política del proceso de integración fronteriza a través de la fijación de orientaciones generales, su transformación en líneas concretas de acción y la movilización permanente en busca de recursos nacionales e internacioales en favor del desarrollo regional.

${ }^{16} \mathrm{~J}$. M. Aragao, "La integración fronteriza como campo de cooperación entre el sector público y el sector empresarial". Documento presentado a la Conferencia Continental del Sector Privado, Ciudad de Panamá, agosto, 1966. 
Finalmente, la inercia observada en diversos sectores de la administración pública, se agudiza en el caso de las zonas fronterizas, alejadas de la sede de la autoridad central, agravándose las deficiencias al tratarse de dos administraciones pertenecientes a jurisdicciones diferentes.

\section{b) Los mecanismos de promoción}

Si bien, en una primera fase aparece como irreemplazable el aporte e interés de los gobiernos centrales en los procesos de integración fronteriza, los esfuerzos gubernamentales ganarían en eficacia y podrán ser atenuados progresivamente, al comprometer paulatinamente los capitales y el interés empresarial privado.

La debilidad o inexistencia de un sector empresario en las zonas de fronteras no es, como padría aparecer a primera vista, necesariamente un indicador de la existencia de ahorros en la región, puesto que diversos estudios y diagnósticos sobre economías retardadas han puesto en evidencia que, pese a su retraso económico y a su relativa pobreza, dichas regiones son exportadoras netas de capital y financian el desarrollo de otras regiones más dinámicas dentro del propio país o aumentan el capital financiero de otros países del mundo.

La insuficiente capacidad de tales áreas para retener los ahorros generados en ellas $y$, con mucha mayor razón, para atraer ahorros desde el exterior, no sólo es debido a los bajos niveles de ingreso, la precariedad de la infraestructura de transportes, de comunicaciones y de energía, sino también al insuficiente conocimiento de las oportunidades de inversión, a la inexistencia de proyectos de factibilidad susceptibles de financiamiento interno o externo y a la inadecuación o inexistencia de los mecanismos de crédito respecto a los objetivos del plan de desarrollo regional.

Este estado de cosas sólo podrá ser corregido con la creación de mecanismos de promoción con atribuciones en el campo técnico y en el financiero $y$, particularmente, con la asignación de recursos para proyectos en el campo de los estudios de preinversión y la preparación de programas y proyectos para las áreas fronterizas.

\section{c) Los problemas cambiarios}

La crisis económica que afecta a todos los países de la región, tiene manifestaciones de inestabilidad particularmente perturbadoras en los niveles de precios de las regiones fronterizas que 
provocan serias alteraciones en el volumen, la composición y el sentido del intercambio intrarregional.

El tratamiento de tales problemas parece que no debe ser sólo enfocado en el campo estrictamente cambiario, sino que deben imaginarse fórmulas basadas en mecanismos compensatorios y de asociación de los intere:es de los sectores pertenecientes a dos zonas limítrofes, hasta tanto se atenúen los impactos que las fluctuaciones cambiarias provocan en la economía regional y se haya logrado una relativa estabilidad en las economías nacionales.

\section{d) La reglamentación del tráfico fronterizo}

Las situaciones de frontera relacionadas con el tráfico fronterizo permiten identificar un obstáculo mayor al intercambio comercial: se trata de la reglamentación del tráfico fronterizo que, por su falta de claridad y su carácter restrictivo, es francamente limitativo a las posibilidades del desarrollo de las áreas limitrofes. Una política de desarrollo y crecimiento productivo, particularmente industrial y agropecuario, es difícilmente concebible si la producción zonal no tiene acceso a los mercados nacionales de los países limftrofes.

\section{e) Las empresas multinacionales}

Aun cuando varios pafses conservan en su legislación prohibiciones severas respecto a la inversión y acceso a la propiedad en sus áreas de frontera, es evidente que una posibilidad que abre interesantes perspectivas es la asociación de esfuerzos y recursos de centros de decisión públicos y/o privados pertenecientes a diversas naciones en la ejecución de proyectos productivos en las zonas limitrofes.

Este tipo de emprèsas multinacionales cuya presencia y actividades deben ser objeto de un contrato que precise su naturaleza y objetivos particulares en función de los requerimientos de la programación del desarrollo fronterizo, podrían constituirse en verdaderos 'centros de planificación conjunta' y de participación comunitaria y su aporte sería particularmente significativo en la modernización de una región determinada y en la formación de polos de desarrollo e integración.

En este enfoque, la empresa multinacional se transforma en un poderoso mecanismo de creación de interdependencias conscientes y deliberadas', con efectos multiplicadores en los diversos países asociados, con proyecciones hacia un desarrollo equilibrado en el sentido geográfico y con sentido más universalista, en la medida en que asocia capitales públicos y privados de diversos 
países y los recursos humanos de otros, en un esfuerzo racional y ordenado de producción y distribución de riquezas'17.

Además de los aspectos anteriormente citados cabe hacer mención de otras modalidades adicionales de cooperación para la integración $y$ el desarrollo fronterizo.

Así por ejemplo, dotar a las regiones fronterizas de una capacidad de análisis económico coyuntrural, parece constituir un requisito importante para evaluar en forma permanente y endógena el impacto sobre la economía de frontera que los modelos nacionales de poítica económica tienen sobre la región, de manera de dar respaldo y racionalidad a los procesos de negociación dirigidos a obtener medidas compensatorias de orden fiscal o de otra naturaleza. Tal capacidad de análisis, concretamente expresada en un centro común de estudios económicos fronterizos, de naturaleza gubernamental o no gubernamental, debe cumplir además un importante papel de apoyo hacia las actividades $y / 0$ proyectos de integración del sector privado, y un no menos importante papel de ayuda en la coordinación binacional de las políticas económicas.

Barros Charlín en su estudio sobre la cooperación económica chileno-argentina plantea otra medida concreta:

"La creación de Corporaciones Regionales de Integración Binacional que promuevan la idea y puedan llevar a cabo proyectos conjuntos y empresas binacionales pueden ser un instrumento eficaz. En esas Corporaciones estarán representados los gobiernos locales y los empresarios; las comunidades locales organizadas, como Corporaciones de Derecho Público podrian tener tratamientos legales y administrativos especiales.

Esas Corporaciones de Integración Binacional podrian entenderse con mayor prestancia institucional y eficacia con la autoridad central. Podrían captar recursos financieros y asesorar a las empresas locales.

Las Corporaciones aludidas tendrían la ventaja de constituir en la región de que se trate, una entidad que centralice esfuerzos; racionalice posibilidades; otorgue prestancia institucional a la región, evitando estructurar una burocracia de indole estatizante.

Sin ánimo de incurrir en mayores detalles concernientes a la estructura jurídica propia de estas Gorporaciones, es útil, por el momento, distinguir dos ámbitos de acción que podrian transformarse en algunas de sus funciones específicas.

${ }^{2 \pi}$ Instituto Internacional de Integración, op. cit. 
i) Nivel de asesoramiento, calificación y promoción en relación a los empresarios regionales:

a) valorar proyectos específicos presentados, aportándoles estudias e identificando oportunidades de inversión en ellos, si fuera del caso;

b) proporcionar asistencia técnica y financiera para preparar y ejecutar proyectos conjuntos o vincular empresas para materializar complementaciones específicas;

c) promover captación y movilización de recursos, pudiendo otorgar fianzas, avales u otras garantías.

ii) Nivel de representatividad:

a) realizar encargos o gestiones especificas encomendadas ante organismos públicos, entidades internacionales, asociaciones empresariales, etc.;

b) representar los intereses regionales aprobados en el Consejo de la Corporación en reuniones binacionales, organismos públicos, eventos nacionales o internacionales de cualquier naturaleza que éstos sean.

La constitución y adecuado funcionamiento de estas Corporaciones Binacionales Regionales de Desarrollo e Integración, enmarcadas en un área geográfica concreta, deben llegax a ser la expresión legal de una regionalización concebida de común acuerdo por los sectores públicos y privados de ambos países. Están destinadas a complementar o a promover, desde la base, auténticas iniciativas de regionalización. No pretenden desplazar ni competir con el sector privado al cual incorporan, ni arrogarse atribuciones ni ejercer presiones indebidas sobre los sectores públicos al cual también incorporan.

La historia de la ya larga lista de convenios y proyectos conjuntos, demuestra que aquellos tratados bilaterales $y$ aquellos esquemas concebidos desde la cúpula, $\tan$ ambiciosos como bien intencionados, caen en el olvido o en el desprestigio, por la "imposibilidad estructural' que tienen de ser capaces de irse adaptando a nuevas circunstancias y desafíos.

El enfoque 'regional' de la complementación económica binacional y de la integración física puede ser menos espectacular polfticamente y aún exhibir una fisonomía fragmentaria, pero emana más establemente de una realidad que suele ser bastante distinta según de qué regiones específicas se trata.

Para recoger ese enfoque regional, las Corporaciones parecen 
ser entes privilegiados, no comprometidos en visiones políticas transitorias ni sujetas a vaivenes diplomáticos"18.

En resumen, la descentralización político-administrativa territorial, la coordinación de las políticas macroeconómicas, la creación de entes binacionales, la concertación pública y privada y la formulación de estrategias y planes de desarrollo fronterizos realmente integrados, pero sobre todo, la clara y persistente voluntad politica de aprovechar positivamente el contacto y la interacción fronteriza, parecen definir el campo de cooperación para la integración.

Las limitaciones existentes a tal cooperación deben ser reconocidas y superadas. Ello supone largos procesos para reestablecer la confianza mutua y para estructurar programas basados en los principios de reciprocidad, compatibilidad y participación, tarea que sería ciertamente facilitada mediante la multiplicación de acuerdos como el Tratado de Paz y Amistad suscrito entre Argentina y Chile en 1984, en el preámbulo del cual se dice que los países lo firman:

"animados del propósito de intensificar la cooperación económica y la integración física de sus respectivos países".

\section{ALGUNOS EJEMPLOS DE GESTIÓN EN REGIONES FRONTERIZAS DE AMÉRIGA LATINA}

Se percibe una gran variedad en la forma de tratar la cuestión fronteriza en los países de América Latina y el Caribe. Sólo de dos países se puede decir que abordan esta cuestión como un asunto de dimensión nacional, en el sentido de intentar un tratamiento orgánico y sistemático de todas las fronteras. En el caso de Chile, el enfoque a la cuestión fronteriza es de naturaleza fundamentalmente política y en consecuencia los instrumentos más destacados son de tipo jurídico y encuentran su máxima expresión en el Tratado de Paz y Amistad entre Chile y Argentina. En el caso de Colombia, el enfoque dado a la cuestión fronteriza es eminentemente económico y desarrollista y su expresión concreta es en consecuencia un Plan Integral de Desarrollo de Fronteras. El resto de los países abordan la cuestión fronteriza de una manera menos integral y en cierto sentido, más casuística.

No siempre el esfuerzo de un país para resolver o manejar sus problemas fronterizos encuentra una adecuada contrapartida en el país vecino. De hecho, en muchos casos se observa más que una acción sistemáticamente concertada entre países, una suerte de "función de reacción" de un país en relación a los programas fronterizos de otro.

${ }^{18} \mathrm{R}$. Barros Ch., op. cit. 
En esta sección y a continuación, se muestran algunos aspectos del manejo fronterizo en algunos países de la región.

\section{a) El caso colombiano}

El Decreto de Gobierno número 3448 emitido en el mes de diciembre de 1983 por el Gobierno colombiano y conocido como el Estatuto de Fronteras, establece en su artículo $\mathrm{v}$ la obligación de preparar un Plan Integral de Desarrollo de Fronteras, obligación que recae en el Departamento Nacional de Planeación. Con tal propósito se identifican como "regiones de frontera" el Departamento Norte de Santander-Gúcuta, César, Nariño-Ipiales, Maicao, Arauca, Casanare, Guajira, Alto Putumayo, Urabá, Chocó, Amazonía, Orinoquía y la isla de San Andres.

El objetivo general del Plan de Desarrollo Integral de las Regiones Fronterizas consiste en asegurar una verdadera integración de las fronteras al desarrollo regional y nacional, que permita superar el estado de marginalidad, atraso y estancamiento económico y social en que tales regiones se encuentran en la actualidad. Subsecuentemente, se propende a un mejoramiento de las condiciones $y$ nivel de vida de la población.

Finalmente se pretende buscar una integración de las fronteras nacionales con las respectivas fronteras de los países vecinos, que vaya más allá del establecimiento de una vinculación de carácter inestable entre tales zonas fronterizas a partir de intercambios comerciales fronterizos, dando paso, entonces a una estrategia de desarrollo común para las regiones fronterizas de países vecinos.

Tanto los elementos derivados de la problemática nacional de desarrollo como los resultantes de la problemática propiamente fronteriza, definen la tipología regional, la cual constituye la base para la formulación de las estrategias de desarrollo fronterizo. En esta perspectiva, la identificación de las estrategias estará guiada por tres objetivos fundamentales, que reflejan tanto las prioridades establecidas en el Plan de Desarrollo del país y Ios Planes Regionales, como las aspiraciones de las zonas de frontera. Los objetivos deberán establecerse según un orden de prioridades definido para las distintas regiones, dado que los mismos tienen un distinto peso y significación según el tipo de regiones de que se trate.

Crecimiento Económico: Este es un objetivo central para aqueIl.as regiones en situación de pérdida de dinamismo, estancamiento o recesión; en las cuales resulta prioritario lograr, en el corto y mediano plazo, una recuperación de la trayectoria de crecimiento, mediante una estrategia de impulso a las ventajas comparativas (agricolas, turísticas y comerciales), y una utilización más plena y eficiente de los recursos naturales, humanos y de capital. La posterior 
Sergio Boisier / Notas en torno al desarrollo de regiones fronterizas...

expansión sostenida de este tipo de regiones dependerá de acciones que fortalezcan y diversifiquen la base económica regional, las articulen más activamente al mercado interno, y refuercen la integración fronteriza.

Equidad: Este objetivo se plantea fundamentalmente para las regiones en transición y con tendencias dinámicas de crecimiento. En estos casos, las estrategias de desarrollo reúnen dos tipos de prioridades: primera, proyectos de vinculación regional a la economía nacional que suponen la presencia estatal para desarrollar la infraestructura física y de apoyo a la producción, así como la conservación y preservación de los recursos naturales; y segunda, programas de servicios públicos básicos dentro de modelos de ordenamiento de las comunidades urbanas que actúan como centros de atracción. En una etapa siguiente, los excedentes obtenidos en las actividades Ifderes deben ser canalizados para asegurar una mayor diversificación de las actividades económicas y una distribución espacial más equitativa de los frutos del crecimiento regional.

Identidad y Soberania Nacional: Este objetivo recae sobre las regiones de más bajo nivel y ritmo de desarrollo. En este caso, la presencia del Estado en forma más activa y sistemática adquiere su mayor prioridad. Sin embargo, las formas que asume dicha presencia tienen características distintas, dependiendo del entorno económico y de la localización de las áreas fronterizas. Pueden identificarse acciones de presencia del Estado que tiendan a fortalecer las comunidades locales, mejorar sus equipamientos colectivos y garantizar una mayor integración intrarregional. En otro sentido, dichas acciones estatales también deben dirigirse a programas de conservación de los recursos naturales para su utilización racional en el mediano y largo plazo, una vez se desarrollen tecnologias no destructoras del medio ambiente.

La implementación del Plan consulta un vasto conjunto de políticas económicas, institucionales y sociales, enmarcadas tanto en el contexto del desarrollo regional como en el contexto de los acuerdos binacionales de integración.

\section{b) El caso chileno}

Como se señaló en el comienzo de este documento, Chile representa un caso peculiar desde el punto de vista del desarrollo fronterizo, puesto que todas las regiones del país son regiones de frontera y todas menos una limitan con Argentina. No es extraño entonces que la frontera argentino-chilena, una de las más extencas del mundo, haya constituido motivo de especial preocupación para ambos países.

El Tratado de Paz y Amistad suscrito entre ambos países el 29 de noviembre de 1984 puso fin a una contienda litigiosa más que cen- 
tenaria, que ha constituido el principal escollo para el aprovechamiento del considerable potencial de integración fronteriza entre ambos países, potencial por lo demás, exhaustivamente estudiado por ambos países.

En el Artículo 12 de aquel Tratado, bajo el título de Cooperación Económica e Integración Física, se dice que:

"Las partes acuerdan crear una Comisión Binacional de carácter permanente con el objeto de intensificar la cooperación económica y la integración física".

La Comisión Binacional estará encargada de promover y desarrollar iniciativas, entre otras, sobre los siguientes temas:

- sistema global de enlaces terrestres.

- habilitación mutua de puertos y zonas francas;

- transporte terrestre;

- aeronavegación;

- interconexiones eléctrica y telecomunicaciones;

- explotación de recursos naturales;

- protección del medio ambiente;

- complementación turística.

El Artículo 12 del Tratado de Paz y Amistad alcanzado por Argentina y Chile, debiera transformarse en el verdadero "resorte de la máquina" de un proceso de paz, basado en una gradual y creciente cooperación e integración económica ${ }^{18}$.

Por otro lado, la Oficina de Planificación Nacional de Chile plantea claramente la indiscutible coincidencia (parcial) en el caso chileno entre el desarrollo regional del país y la integración fronteriza:

"Al realizar un análisis del país según sus ejes transversales se visualiza el hecho de que Chile cuenta con numerosos pasos y rutas transcordilleranas que interconectan algunas regiones productivas muy próximas $y$ que han mantenido un intercambio histórico casi sin interrupción. Este intercambio actualmente presenta magnitudes débiles en comparación con el intercambio interno de cada uno de los países, pero se vislumbra un nuevo giro que debería retomar e incrementar las proyecciones históricas, debido a diferentes factores.

Frente a este hecho próximo, que ya comienza a manifestarse ${ }^{19}$ Ibid. 
Sergio Boisier / Notas en torno al desarrollo de regiones fronterizas...

con contactos y acuerdos bilaterales entre regiones fronterizas, conviene reforzar la visión de planificación que vela por los intereses del conjunto de regiones. Vale decir, por el nivel nacional-regional de planificación y decisión, ya que la apertura directa de la integración puede significar un perjuicio para regiones no participantes, o bien, un posterior descontrol en el intercambio si éste no es equitativo en términos globales de economía nacional; lo que, por otro lado, puede derivar en una frustración, por décadas, de la aplicación misma de este incentivo del desarrollo: la integración ${ }^{20}$.

Según lo describe Barros 'Charlín en su trabajo ya citado, el marco bilateral de las relaciones entre Chile y Argentina entre 1958 y 1978 registra: a) la creación de Comisiones Permanentes; b) la formulación de Declaraciones Politicas y; c) la suscripción de Convenios y Acuerdos Bilaterales. Muchos de estos referentes tienen relación directa con la integración y el desarrollo fronterizo especifico.

\section{c) El caso de la frontera boliviano-peruana}

La región fronteriza de Bolivia y Perú tiene una línea de contacto de alrededor de $1.100 \mathrm{Kms}$. de extensión y una superficie total de aproximadamente $363.363 \mathrm{Kms}^{2}$, correspondiendo $197.812 \mathrm{Kms}^{2}$ a Bolivia y $165.551 \mathrm{Kms}^{2}$ a Perú, e incluye una población de 2.715.167 habitantes $(61,5 \%$ boliviana).

En el estudio del Instituto Internacional de Integración se señala lo siguiente en relación a esta región:

"En el marco de la gran cantidad de convenios bilaterales existentes entre Bolivia y Perú, aquellos referidos a las actividades vigentes entre ambos países y que contienen componentes de integración fronteriza serían los siguientes:

1. Proyecto Piloto sobre Cultivo de Truchas en jaulas flotantes, que estuvo manejado por Pesca Perú y cordepaz; actualmente se busca un acuerdo que permita crear un organismo que represente el proyecto conjunto.

2. Plan de Evaluación Pesquera del Lago Titicaca y su cuenca, que está representada por el Instituto del Mar, del Perú (IMARPE), y por la Universidad Mayor de San Andrés (UMSA), por Bolivia.

3. Promoción del Desarrollo en el Área Fronteriza bolivianoperuana; es un trabajo que ha sido encomendado a las cor-

${ }^{20}$ Oficina de Planificación Nacional, Chile, "Estrategia Nacional de Desarrollo Regional 1975-1990", Santiago de Chile, 1976. 
poraciones de desarrollo de Puno y La Paz con fondos financiados por la Corporación Andina de Fomento (CAF).

4. Decisión $141 \mathrm{dt} / 185$ (JUNAC) Programa Acción Conjunta Perú.Bolivia, referido a dotar de facilidades de transporte a Bolivia debido a su condición de país mediterráneo.

5. Implementación decisiones 56 y $56 \mathrm{~A}$ (JUNAC). Otros convenios más especializados y localizados (ejem.: para. el control de la fiebre aftosa), también han tratado de ser implementados, con escaso éxito. Entre los anteproyectos se encuentran:

a) Convenio sobre tránsito de personas.

b) Grupo Mixto de tránsito de personas.

c) Constitución de un complejo fronterizo en Kasani.

6. Decisión 185 JUNAc. Que contempla la aprobación de un Plan Andino de Acción Conjunta para facilitar el tránsito de Bolivia hacia puertos peruanos.

Por otra parte, en la esfera de las agencias creadas para tratar especialmente materias fronterizas, existen dos comisiones mixtas entre los gobiernos de Bolivia y Perú:

a) La Comisión Mixta Permanente Peruano-Boliviana de Coordinación (COMTCOORD), creada el 18 de febrero de 1971.

b) Comisión Mixta Boliviano-Peruana para el Desarrollo Conjunto del Altiplano (COMIDALT), creada el 13 de septiembre de 1974.

La implementación de los Acuerdos mencionados y el avance en las negociaciones tendientes a facilitar los intercambios entre ambos países han sido lentos. Sin embargo, la voluntad política, unida a un ajuste de los mecanismos existentes permitiría desarrollos significativos en la integración fronteriza boliviano peruana" 21 .

\section{d) El caso de la frontera mexicano-norteamericana}

La frontera entre México y los Estados Unidos representa probab!emente el caso más complejo de entre todas las "situaciones fronterizas" de América Latina. No sólo es una de las más extensas en longitud; adicionalmente en ambos lados de la frontera se ha desarroilado un conjunto de centros urbanos en ráp:da expansión y que mantienen relaciones simbióticas entre ellos. Todavía más, en el

mInstituto Internacional de Integración, op. cit. 
Sergio Boisier / Notas en torno al desarrollo de regiones fronterizas...

lado mexicano se ha desarrollado bajo impulso oficial toda una vasta estructura industrial de sub-contratación internacional (maquiladoras), con importantes efectos económicos y sociales sobre la frontera. Como si esto fuese poco, se ha generado toda una "cultura fronteriza" en esta zona, expresada en la literatura, en la música popular $y$ en el cine. Es evidentemente, una frontera viva.

En 1980 la población de las principales ciudades a ambos lados de la frontera era la siguiente:

\begin{tabular}{lclr}
\hline Ciudad & Población (1.000) & Ciudad & Población (1.000) \\
\hline Tijuana & 542 & San Diego & 1.861 \\
Mexicali & 495 & Tucson & 531 \\
Ciudad Juárez & 680 & Las Cruces & 96 \\
Nueva Laredo & 272 & El Paso & 480 \\
Reynosa & 240 & Laredo & 99 \\
Matamoros & 258 & McAllen & 283 \\
& & Brownsville & 210 \\
\hline Total México & 2.487 & Total u.s.A. & 3.560
\end{tabular}

Todas estas ciudades han estado expandiéndose en la década de los 70 a tasas mucho más elevadas que las tasas nacionales de crecimiento demográfico en los Estados Unidos y en México.

Más aún, si bien el nivel de vida en las ciudades fronterizas mexicanas es relativamente bajo de acuerdo a los estándares americanos, de todos modos es más elevado que en el resto de México. Por ejemplo, según lo comenta Hansen, el salario mínimo varía en México entre los estados así como entre sub-regiones dentro de los estados. Las sub-regiones de la frontera muestran tasas salariales reIativamente altas; Baja California exhibe la tasa salarial más elevada de todo México. Aún así, las ciudades fronterizas mexicanas sirven como base para muchas personas que trabajan en el lado norteamericano, sea como "commuters" Iegalmente reconocidos o simplemente como "indocumentados". Por otro lado, muchas empresas norteamericanas han sido atraídas hacia el lado mexicano de la frontera por medio del "programa de maquiladoras" del Gobierno mexicano. Bajo tal arreglo, las subsidiarias mexicanas de corporaciones americanas arman productos cuyas partes originales han sido fabricadas en los Estados Unidos y en seguida exportan tales productos a los Estados Unidos pagando aranceles sólo sobre el valor agregado, es decir, sobre mano de obra de bajo costo. Las "maqui- 
ladoras" proveen alrededor de 130.000 empleos a mexicanos de la frontera ${ }^{22}$.

Entre 1966 y 1976 el número de empresas maquiladoras en la frontera se elevó de 25 a 448, con una fuerte concentración en la fabricación de máquinas, aparatos, accesorios y artículos eléctricos y electrónicos. El efecto empleo de estas empresas es importante, pero es necesario calificar cuidadosamente algunos efectos sociales negativos producidos por las maquiladoras ${ }^{23}$,

Desde el punto de vista de un desarrollo concertado de la frontera, Hansen señala que con excepción de la Comisión sobre Agua y Límites Internacionales, los intentos de los gobiernos centrales de ambos países para enfrentar los problemas fronterizos han sido inestables y poco efectivos y sin duda, desde el punto de vista de la percepción local, frecuentemente han sido contraproducentes. Como lo indicara un habitante encuestado en Laredo: "Aquí en Laredo vivimos peligrosamente. Estamos expuestos a las inundaciones, a las sequías, al Gobierno mexicano y al Gobierno norteamericano".

e) El caso de la frontera argentino-uruguaya ${ }^{24}$

"En esta frontera existe un aprovechamiento binacional, la central hidroeléctrica de Salto Grande, cuya construcción fue llevada a cabo por una Comisión Técnica Mixta que también se encarga del mantenimiento y la administración. La Comisión 'Técnica Mixta, con la asistencia técnica del BID, diseñó un plan de desarrollo regional para el área de influencia de la represa que comprende en el lado argentino, los departamentos de Colón, Concordia y Federación, pertenecientes a la provincia de Entre Ríos, y el departamento de Montecaseros, de la provincia de Corrientes. En el lado uruguayo se consideraron los departamentos de Artigas y Paysandú, en una franja de 70 kilómetros de ancho que bordea el río Uruguay. En el estudio del plan se advierte que 'se trata de hecho de dos economías regionales poco relacionadas entre sí, y cada una de ellas tiene un escaso nivel de integración en sí misma'. El plan de desarrollo regional se diseñó para paliar los efectos negativos de la construcción de la central y, a la vez, con el fin de conformar un área de integración binacional. Para el corto plazo se identificaron proyectos de inversión repartidos en cada área nacional, con dos grandes pro-

$\approx$ N. Hansen, op. cit.

${ }^{3}$ A. Levy y S. Alcocer, Las maquiladoras en México (México: Fondo de Gultura Económica, 1983).

atEste comentario se ha extraido de: Bolognesi-Drosdoff, M. G. (1986), "Anílisis y clasificación tipológica de casos de integración fronteriza", Integración Latinoamericana, año II, No 118, Intal, Buenos Aires, noviembre 1986. 
yectos industriales, una planta de urea y otra de ferroaleaciones, que requerirían la formación de empresas binacionales. Para el mediano y largo plazo, se recomendó mantener un sistema de monitoreo de tendencias destinadas a ampliar la base de información, diversificar la producción, generar capacidad empresarial y usar los recursos naturales en forma eficiente. Se recomendó la formación de empresas b:nacionales para ampliar mercados; la comercialización conjunta cuando se trata de exportar a terceros países, y la regulación conjunta de la explotación pesquera y el turismo.

Se propuso, asimismo, un Programa de Asistencia Técnica y Crédito Orientado (PATCO), como estructura organizativa permanente dentro de la Comisión Técnica Mixta, con funciones de promoción, coordinación y monitoreo. De cumplirse este plan, se estima que su repercusión en la economía regional sería mayor que la derivada de la construcción de la central.

En esta frontera existen tres ejes de intercambio a través de puentes sobre el río Uruguay. Dos de ellos están en la zona de influencia de Salto Grande: Salto-Concordia y Paysandú-Colón; uno, al sur Fray-Bentos-Puerto Unzué. En éstos se aplica el acuerdo relativo al control único de frontera y de documentación unificada. En las fronteras se entrega una tarjeta de habilitación para el tráfico vec.nal fronterizo, válida por tres años, que se puede obtener mediante la presentación de una solicitud, documento de identidad y certificado de domicilio. Esta tarjeta permite la libre circulación entre las localidades fronterizas".

\section{f) El caso de la frontera brasilera-paraguaya}

Entre los casos de situaciones fronter:zas existen algunos que se asimilan más al concepto de zonas de integración fronteriza, o de desarrollo compartido. Es el caso de la frontera entre Brasil y Paraguay sobre el río Paraná. EI mismo estudio del INTAL recién citado señala con respecto a esta frontera:

"Esta frontera se extiende en aproximadamente 200 kilómetros a lo largo del Paraná, desde los Saltos del Guairá hasta la confluencia del río Iguazú. En Brasil, incorpora la zona al oeste del estado de Paraná, y en Paraguay los departamentos de Alto Paraná y Canendiyú. Desde el punto de vista socioeconómico es un área sujeta a migraciones recientes y a recambio de cultivos. Las migraciones se orientaron hacia el Alto Paraná paraguayo, desde el vecino país, y desde el interior de Paraguay.

Existen dos ejes de intercambio entre los dos países, el de Guairá, al norte, y el más importante, Foz do Iguazú y Giudad 
Presidente Stroessner. C'udad Presidente Stroessner se ha convertido en el segundo centro urbano de Paraguay en cuanto a actividad económica y número de hab tantes. Esta es una frontera relativamente abierta, con gran movilidad de factores. También es un área donde se aplicaron acciones y proyectos de desarrollo con repercusión en ambos lados de la frontera. El proyecto binacional de Itaipú contribuyó en gran medida a la transformación económica operada en la zona, a pesar de los efectos recesivos que sobrevinieron a su terminación. El proyecto ha dado lugar a programas sanitarios, de salud, educación, obras de infraestructura vial y urbana y de conservación del medio ambiente. También se impulsó la actividad turística.

Esta frontera presenta características propias determinadas por la extensión de la frontera agrícola y la construcción de Itaipú. En los últimos 25 años, estos dos hechos originaron una transformación económica muy considerable en la zona, que le da una cierta homogeneidad para propósitos de estudios y para la posible aplicación de políticas conjuntas, de tal modo que se podria considerar una zona de integración regional fronteriza.

En la actualidad, la región que constituye el área de influencia de la represa de Itaipú es agroexportadora y estuvo sujeta a grandes cambios estructurales en relativamente pocos años.

El departamento de Alto Paraná experimentó una transformación agrícola notable. Nuevos cultivos comerciales se introdujeron junto con la mecanización agrícola producto del asentamiento de colonos del vecino estado brasileño de Paraná. En el lado brasileño, en el estado de Paraná, entre 1962 y 1972 se introdujo un cambio en el tipo de cultivos: del café al trigo y la soja, lo que originó una reestructurarión económico-social.

Diferenciales en el precio de la tierra y condiciones de suelo muy similares atrajeron a la población brasileña al Alto Paraná paraguayo. Esta atracción creció porque en el lado paraguayo los impuestos eran inexistentes y si se disponía de poder adquisitivo, el acceso al crédito era más fácil. El Gobiemo paraguayo, por su parte, facilitó la colonización brasileña al levantar todas las restricciones legales a la compra de tierra por extranjeros. La represa de Itaipú, al inundar tierras del lado brasileño, aceleró la inmigración hacia el país vecino.

La otra gran transformación que se produjo en este espacio geográfico se debió a la construcción de la represa de Itaipú. El mayor impacto se manifestó en la urbanización de Foz do Iguazú-Giudad Presidente Stroessner. En 1979, considerado año pico en la actividad de construcción, en el ente binacional creado para hacerse cargo del proyecto Itaipú Binacional, la población au- 
mentó de 20.000 habitantes en 1970 a casi 100.000 en 1978 , en tanto que Ciudad Presidente Stroessner pasó de 10.000 habitantes en 1972 a más de 73.000 en 1982. Si se tienen en cuenta que ambas orillas están interrelacionadas y que es relativamente fácil pasar de una ciudad a otra a través del puente internacional, se podria hablar de un área urbana fronteriza que en 1980 ya contaba, aproximadamente, con 170.000 habitantes.

El sector de la construcción fue el que más creció. Surgieron también industrias conexas, pero a pesar de los esfuerzos de los gobiernos para constituir una base industrial estable, muchas de estas plantas cerraron cuando finalizó la obra. La terminación de las obras de Itaipú trajo aparejada la recesión en el área. Hacia 1983, el nivel de empleo que se había mantenido gracias a la construcción de la represa, se redujo considerablemente.

En el espacio geográfico delimitado por la zona de influencia de Itaiprí, se generaron y profundizaron interrelaciones socioeconómicas y políticas que representan cambios considerables en la estructura productiva de la región. El proceso de integración fronteriza por el intercambio de factores y aprovechamiento de los recursos naturales ha producido beneficios para ambos pafses, tanto a nivel nacional como de la región"2s.

CONGLUSIÓN: LA FRONTERA COMO FACTOR DE INTEGRACIÓN ${ }^{26}$

Distintos autores se han referido a América Latina describiéndola como un territorio fragmentado en el que las fronteras interrumpen la natural continuidad de la región. Esta afirmación es comprobable fácticamente aun en la actualidad, y pese a los avances que se han verificado principalmente en el campo de la integración. Es a partir del decenio de 1920 que se comienzan a habilitar en forma importante conexiones de transportes y comunicaciones, y sólo de modo más o menos generalizado e intenso a partir del decenic de 1950, coincidentemente con el aumento del comercio intraregional.

${ }^{30}$ M. C., Bolognesi-Drosdoff, "Análisis y clasificación tipológica de casos de integración fronteriza", Integración Latinoamericana, año II, No 118, INTAL, Buenos Aires, noviembre 1986.

mparte del texto del Editorial del número 138 de la revista Integración Latinoamericana, revista mensual del INTAL, publicada en Buenos Aires, República Argentina, noviembre de 1986. 
El modelo de crecimiento de la economía de los países latinoamericanos, basado en la exportación a terceros países, definió una conformación espacial de la producción y de la población que en general no ocupaba áreas interiores y fronterizas de los países. En diversas oportunidades estas fronteras fueron escenarios de conflictos de soberanfa, lo que originó como respuesta correctiva la ocupación mediante asentamientos humanos diseñados en función de una polftica nacional y no de una estrategia de desarrollo fronterizo que armonizara intereses y acciones de los padses limitrofes.

La frontera, así concebida como un elemento restrictivo y diferenciador, se convirtió en el área de recolución "sui generis" de las contradicciones polfticas y económicas de los países limítrofes. Su funcionamiento, en oportunidades desordenado y hasta caótico, origina tensiones frecuentes: el contrabando, la migración ilegal, el entorpecimiento del transporte y los conflictos en el uso de los recursos fronterizos convivieron con otra realidad, cual era la apari"ón de poblaciones estables, adaptadas al estilo propio de la vida de la frontera, que comerciaban, establecían relaciones sociales, utilizaban servicios de uno y otro lado de la frontera, aún al margen de los instrumentos reguladores.

Frente a esta "realidad fronteriza", más fuerte cuanto mayor es la diferencia entre las economías colindantes, principalmente en precios relativos e ingreso, aparece contemporáneamente la necesidad y conveniencia de regular la actividad fronteriza y tratar de intervenir positivamente creando mejores condiciones para un ordenamiento y desarrollo complementario de las áreas limítrofes. Surgen así los primeros intentos por aplicar políticas específicas en la fron. tera colombo-venezolana, en la argentino-boliviana, a lo largo de la frontera del Brasil, etc., y también las experiencias de armonización y de desarrollo conjunto.

En la medida que se armonicen políticas comerciales y cambiarias disminuirán ciertas tensiones en la frontera, originadas principalmente por la diferencia de precios relativos, pero se aumentará la necesidad de obtener un comportamiento eficaz en materia de transporte, aduanas $y$, en general, de lo que ha dado en llamarse globalmente facilitación.

Sin embargo, el enfoque de la frontera requerirá no sólo abordar los aspectos regulatorios sino también aprovechar los factores de localización propios de la frontera, como la combinación de recursos fronterizos incluyendo el mercado para promover su desarrollo armónico y conjunto.

La frontera debe ser atendida como una posibilidad adicional de articular las economías de los países y de mejorar la propia orga-

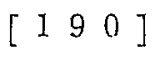


Sergio Boisier / Notas en torno al desarrollo de regiones fronterizas...

nización del espacio económico de cada uno de éstos. En ambos sentidos, la acción debe ser simultánea con aquellas otras destinadas a promover la integración comercial e industrial, teniendo en cuenta, sin embargo, la sensibilidad del hecho fronterizo, que, de no ser manejado adecuadamente, encierra un potencial de conflicto capaz de interferir negativamente la relación bilateral. 